\title{
Multipath Routing Protocol using Cross-layer based QoS Metrics for IEEE 802.11e WLAN
}

\author{
Shaik Madhar Saheb \\ Caledonian College of Engineering \\ PO BOX 2322 \\ CPO Seeb 111, Oman
}

\author{
A. K. Bhattacharjee \\ Department of Electronics and \\ Communication Engineering, NIT, \\ Durgapur, India
}

\author{
Dharmasa \\ Caledonian College of Engineering \\ PO BOX 2322 \\ CPO Seeb 111, Oman
}

\begin{abstract}
To achieve Quality of Service (QoS) support for multimedia traffic, IEEE 802.11e specifies Hybrid Coordination Function (HCF) Controlled Channel Access (HCCA) technique. When it is applied in multi-hop networks, the performance results, however, reveal that there is significant degradation of throughput with high delay at high load conditions. This is due to the fact that routing is responsible for the successful packet delivery and QoS Support. In a single path routing protocol, although multiple paths are discovered by the source during the process of discovering a route, the path with the shortest delay is chosen as the best path while discarding the rest. An increased efficiency can be achieved in terms of QoS metrics, balancing the load and tolerance to faults by the use of multipath routing. Apart from using multipath routing, cross-layer based approach is also necessary for the enhancement of QoS in IEEE 802.11e networks. This paper proposes a multipath routing protocol using cross-layer based metrics for improving the QoS of multimedia traffic in IEEE 802.11 e networks. Initially a combined cost metric is formed based on traffic contention time, average transmission delay and signal fading values. The path with minimum combined cost is chosen as the primary path from the multiple disjoint paths discovered. In case of failed data transmission, the traffic is re-routed through an alternate path with next minimum cost. Simulated Performance evaluation substantiates the reliability of the proposed protocol in achieving higher delivery ratio and throughput and reducing the delay.
\end{abstract}

\section{General Terms}

Average Transmission Delay, Multipath Route Discovery Process, Signal Fading Value, Traffic Contention Time.

\section{Keywords}

Access Point, Basic Service Set, Combined Cost, CrossLayer, Multipath Routing, Traffic Class.

\section{INTRODUCTION}

Wireless Local Area Network (WLAN) designed by IEEE 802.11 has Basic Service Sets (BSS) containing wireless stations (STA). IEEE 802.11 WLAN stipulates a Distributed Coordination Function (DCF) and a Point Coordination Function (PCF). It possesses an autonomous BSS along with an infrastructure network. This network containing an access point (AP) and its associated STA's can be formed as ad hoc network. In a BSS, the coordination functions control the channel access of the STA [1]. These technologies are widely deployed as the most preferred wireless access technology in the recent past. There are natural limitations of wireless technologies that block the communication. Here the quality of service features of the 802.11e standard is advantageous to prioritize. Examples include Voice and video traffic over more elastic data traffic [2]. Though data applications are offered by IEEE 802.11 there is no QoS support for multimedia applications. To improve the QoS support for WLAN applications, IEEE 8202.11e standard stipulated a set of QoS enhancements [3]. It specifies the Hybrid Coordinator (HC) located in QoS Access Point (QAP) which controls the HCF. The Enhanced Distributed Channel Access (EDCA) and the HCCA specified by HCF define Traffic Classes (TC) providing service differentiation. The HCCA is effective only in handling data streams with constant bit rate (CBR) traffic and does not overlap with QoS Base Service Sets (QBSSs) and therefore should be improved [4]. Notwithstanding the traffic conditions, the HCCA method of 802.11e standard makes parameterized QoS support available, reducing the need for carrier sensing and backing off during the controlled access phase. Replacement is made based on a pre-computed schedule. A scheduler residing at the AP controls entire access to medium within the BSS and polls member stations for transmissions [5]. HCF is used as a method for control based channel access. QoS requirements are satisfied once a stream is admitted to the network. Resource allocation is managed by the HCCA mechanism using the HC located at the AP which supports the QoS facility [6]. HC has the first preference in a wireless medium access as it can start transmission for a very short time interval when it senses the channel being idle [7]. HCCA and PCF work in a similar fashion. The medium is controlled by AP for one period, when the beacon frames interval is separated whereas, channel members along with EDCA control the medium during the next period. In PCF, the traffic is split into classes. The HC coordinates with these classes and schedules one station over the other on priority basis by collecting the queue length of the traffic from the stations [8]. With HCCA better QoS accuracy can be achieved. Advanced applications like VoIP and video streaming work more efficiently on a Wi-Fi network because of the ability of QoS-enabled stations to acquire specific transmission parameters such as data rate, jitter and so on [9]. Considerable degradation in throughput and delays at high load conditions can still be noted in HCCA, notwithstanding various enhancements made to achieve QoS support. This can be attributed to the increase in time to negotiate the channel access and transit network behaviour. For application delivery and QoS support the route quality is vital [10]. To maintain overall QoS satisfaction, it is very crucial to have an effective admission control in the 802.11e networks. Proper admission decision can only be made if routing protocols incorporate QoS requirements of flows at the end-nodes in the network. 
Most routing schemes do not provide distinct QoS support as they attempt to get the shortest possible path. An efficient distributed admission control can be designed only when there is a QoS routing support which is required to discover routes with less interference [11]. The Media Access Control (MAC) layer and routing protocols are mutually affected by each other. A virtual network is triggered by the paths taken by the routing protocols when at a particular node the MAC protocols are stimulated. The initiation of new route queries and updates to the routing table, however, leads to contention at the MAC layer [12]. In single path routing protocol such as Ad hoc On-Demand Distance Vector (AODV), although multiple paths are discovered by the source in the discovery process, only the route with the shortest possible delay is chosen while discarding the rest. This leads to recurrent route breaks as there is no alternate path to destination and hence packets are dropped by the intermediate nodes. Multi-path routing has definite advantage over single path routing protocols in that the traffic is distributed among all several available good paths rather than only the best path available. In doing so there is a significant improvement in the network utilization and by diverting traffic to unused network resources, proper load balancing is provided, thereby reducing congestion in hot spots. Multi-path routing helps to reduce performance issues and excessive variance in congested links by providing steady and smooth data streams through fault tolerance, load balancing, bandwidth aggregation, and improvement in QoS metrics such as delay [13]. However, the IEEE $802.11 \mathrm{e}$ and multi-path routing protocols interaction is further to be researched. The cross-layer design approach uses multilayer parameters from Open Systems Interconnection (OSI) stack in order to enhance the performance of multi-hop wireless networks such as Wireless Sensor Networks (WSN), Mobile Ad Hoc Networks (MANET), WLAN as well as Wireless Mesh Networks (WMN) [14]. In the design of a WLANs protocol, criteria such as physical layer adjustment to the rapid changes in link characteristics, minimizing collision, ensuring fairness in the access of MAC layer and a routing decision to effectively deliver data to the destination, have to be met by the network layer. However, the layered protocol design methodology does not always provide optimum solution for dynamic environment. With layered protocols energy cannot be regained from WLAN routing protocol. It is also not possible to fetch location information or to calculate routes from the underlying data link and physical layers. Hence, a cross-layer protocol design is indispensable to enhance the WLANs performance. The organization of the paper is as follows: Section 2 discusses related work. Section 3 describes proposed solution. In section 4 simulation results and discussions are presented and finally conclusions are presented in the section 5 .

\section{RELATED WORK}

All Lamia Romdhani et. al. [10] proposed a routing protocol based on Cross Layer metrics, CLAE, for finding out the best possible path including the parameters such as transmission delay, network bandwidth, and stability of route and so on. CLAE is developed based on the communication properties of On-Demand AODV and IEEE 802.11e MAC (EDCA). Their focus was mainly on establishing the path with lesser delay and without loss of generality. Their buffer management scheme developed for audio class service aims to reduce undesirable network behavior (resulting from the setup of new channels of communication and node mobility), and to distinguish audio packets based on their tolerated end-to-end transfer delay and current experienced delay. Ron Banner et. al. [13] have adopted a meticulous approach to investigate the multipath routing and established the considerable benefit of multipath routing in providing best possible solutions. Noureddine Kettaf et. al. [15] have suggested a cross-layer routing protocol for mobile ad hoc networks based on the interaction between Admission Control enabled On-demand Routing (ACOR) protocol and QoS-based IEEE 802.11e MAC layer. The aim of their protocol is to find a viable route in line with the application QoS requirements. Víctor Carrascal Frias et. al. [16] have put forward a QoS-aware multipath Dynamic Source Routing (DSR-based) routing protocol to enhance the connection reliability while load balancing and reducing the end-to-end delay. Carlos $\mathrm{T}$. Calafate et. al. [17] have extensively studied the results of the interaction between reactive routing protocols for MANETs and the IEEE 802.11e standards and found that considerable enhancements in throughput and normalized routing overhead can be achieved due to the increase in the responsiveness of routing. Multipath Routing Protocol (CMRP) based on Crosslayer design for mobile ad hoc networks propounded by Hang Shen et. al. [18] uses the means of the node energy signal from the physical layer. This protocol aims to optimize routing decision and quality of path.

\section{PROPOSED PROTOCOL}

\subsection{Overview}

The protocol proposed here at first calculates multiple disjoint paths and then estimates the Combined Cost (CC) metric based on the metrics such as: Traffic Contention Time (TCT), Average Transmission Delay (ADelay) and Signal Fading Value (SFV). Finally, the path with minimum cost (i.e., with minimum (C) is chosen as the best path from the multiple disjoint paths and the data is sent through.

\subsection{Estimation of Traffic Contention Time Metric}

In the beginning, A MAC layer throughput model which provides an upper bound for shared wireless network with nodes having varied rates of transmission is defined in accordance with the IEEE 802.11 standard. Extensions to IEEE 802.11e HCCA MAC protocol are defined when nodes gain access to the channel in their transmission opportunity (TXOP) and are permitted to keep it for a specified interval of time. Finally, the Traffic Contention Time (TCT) metric is defined. The transmission throughput upper bound for the basic Carrier Sense Multiple Access with Collision Avoidance (CSMA/CA) and the Request to Send / Clear to Send (RTS/CTS) mechanism is given as,

$$
A T=\frac{P L_{k}}{\sum_{j=1}^{N} \frac{P L_{j}}{R_{j}}}
$$

where,

AT - the Average Throughput, $\mathrm{PL}_{\mathrm{k}}$ - the Packet Length for node $\mathrm{k}, \mathrm{R}_{\mathrm{k}}$ - Rate.

To deal with classes of multiple weighted services, equation (1) can further be extended. In a class, the throughput attained by a flow and the class weight are proportional. The 802.11e's HCCA standard is the best example that supports various values of minimum contention window. To achieve an average throughput in HCCA, it must be proportional to the class weight and is inversely proportional to the minimum value of the contention window. In addition, when contention level is not more, the probability of a node's attempt is 
inversely proportional to minimum value of the contention window.

Thus, if $W_{i}$ is weight of the node $\mathrm{i}$, then equation (1) in the case of 802.11 e HCCA can be given as,

$$
A T=\frac{W_{k} P L_{k}}{\sum_{j=1}^{N} \frac{W_{j} P L_{j}}{R_{j}}}
$$

The time estimate for a packet to be transmitted through link $l$ is $P L_{l} / A T_{l}$, where $A T_{l}$ will be as (2). Therefore, the $T C T$ metric of a weighted service differentiation can now be given as,

$$
\text { TCT }_{l}=\frac{1}{W_{l}} \sum_{j \in N_{l}} \frac{W_{j} P L_{j}}{R_{j}}
$$

\subsection{Estimation of Delay Metric}

The performance of an application is impacted by the MAC layer as is evident from various research works. The proposed scheme saves the time at which the unicast packet arrives when it is received from the routing layer by the MAC layer which will then be forwarded to the next hop. Similarly, it calculates the delay in the transmission when the acknowledgment (ACK) is received. This can be given as,

$$
T_{\text {delay }}=T_{\text {ack }}-T_{\text {rec }}
$$

where $T_{a c k}$ is the time at which the ACK is received by the MAC layer and $T_{r e c}$ is the time at which a packet is received from the routing layer.

For all the transmitted packets, $T_{\text {delay }}$ is calculated for a tunable period $T$. In case of less or no traffic the transmission delay is taken as it is. Taking the average delay in transmission at the end of every period $T$, the node updates the respective priority. In addition, the average time of transmission is estimated only when the packets are successfully sent in intermediate nodes. The ratio of the packet delay calculated at step $j$ initially is noted by $D_{\text {curr }}^{j}$. To minimize transient delay bias, an estimator of Exponentially Weighted Moving Average (EWMA) is used to smooth the values estimated. Assuming that for each period of update $T$ at step $j$, the average delay $A_{\text {Delay }}^{j}$ is computed iteratively as given below,

$$
A_{\text {Delay }}^{j}=(1-\lambda) \times D_{\text {curr }}^{j}+\lambda \times D_{\text {Delay }}^{j-1}(4)
$$

where $j$ is the jth period updated $T, D_{\text {curr }}^{j}$ the current delay, and $\lambda$ the leveling value which determines the size of memory used in calculating the averages.

\subsection{Estimation of Signal Fading Value Metric}

Wireless communication is characterized by severe signal fading (SFV). SFV helps in predicting the mobility of the node. The bigger the SFV, the higher is the distance and hence the higher the probability of link interruption. Hence, the path with low SFV is considered stable as it is energy efficient which in turn can reduce signal attenuation for packets sending and therefore, data packets can be transmitted through this path.

In equation (5) SFV, transmitted by routing message, of the node is defined. This indicates the amount of stability between two serial nodes relative to the entire path,

$$
S F V_{i}=\frac{R E_{i}-C E_{i}}{C E_{i}}
$$

where $R E_{i}$ is the remaining node energy for the node $i, C E_{i}$ the fixed energy consumption for node $i$ of every efficient data packet sending.

\subsection{Multipath Route Discovery Process}

\subsubsection{Route Request (RREQ)}

Using the equations (3), (4) and (5), the TCT, $A_{\text {Delay }}$ and $S F V$ can be estimated for each node respectively. The combined cost then is determined as,

$$
(C C)=\frac{a^{*} T C T+b^{*} A_{\text {Delay }}}{c^{*} S F V}
$$

where $\mathrm{a}, \mathrm{b}$, and $\mathrm{c}$ are normalization or smoothing constants. Let us suppose a source $S$ needs to send data to the destination node $D$ through the intermediate nodes $R 1, R 2$ and $R 3$. To initiate discovery of QoS-aware route, an $R R E Q$ is sent by the source $S$. When the $R R E Q$ packet is received by $R 1$, it estimates the metrics mentioned earlier.

Firstly, the host $R l$ calculates its cost $C C R l$ using equation (6).

$$
R R E Q_{R I}======\rightarrow R 2
$$

Secondly, $R 2$ calculates its cost $C C R 2$ and adds it to the cost of $R 1$. Then $R 2$ forwards the $R R E Q$ including the added cost.

$$
\begin{gathered}
C C_{R 1}+C C_{R 2} \\
R R E Q_{R 2}===========\Rightarrow R 3
\end{gathered}
$$

Lastly, when it reaches the destination node $D$, the $R R E Q$ will contain all the combined costs of the nodes.

$$
\begin{gathered}
C C_{R I}+C C_{R 2}+C C_{R 3} \\
R R E Q_{R 3}==============\Rightarrow D
\end{gathered}
$$

\subsubsection{Route Reply (RREP)}

The RREP packet with the combined costs of node, is sent by the destination node $D$ to the nearest upstream node $R 3$.

$$
\begin{gathered}
C C_{R 1}+C C_{R 2}+C C_{R 3} \\
R R E P============\rightarrow R 3
\end{gathered}
$$

$R 3$ computes its Combined Cost $C C$ from $R R E P$ which can be given as,

$$
C C=\left(C C_{R 1}+C C_{R 2}+C C_{R 3}\right)-\left(C C_{R 1}+C C_{R 2}\right)
$$

Similarly, all the intermediate hosts calculate their costs. The route with least cost value is selected by the source from the total RREP it receives. In case of failure or breakage in any route in the transmitted path, it selects the next available best path from the multiple disjoint paths and reroutes the data through that path.

\section{RESULTS AND DISCUSSION 4.1 The Parameters of the Simulated Model}

NS2 [20] is used to replicate the suggested mechanism and implemented over the enhanced 802.11e WLAN HCCA scheduler [19]. The channel capacity of mobile hosts is set to 
a constant value of $11 \mathrm{Mbps}$. In the simulation time of 50 seconds, 10 Quality of Service aware Stations (QSTAs) and a base station (BS) are installed in a $1000 \times 1000$ metre region. Each node has a 250 metre transmission range. TCP upload traffics are sent from wireless stations. The proposed Multipath Routing protocol with Cross-layer Based QoS metrics (MRCBQ) is compared with CLAE [10] protocol.

Table 1. Gives a summary of the simulation settings and parameters

\begin{tabular}{|l|l|}
\hline Area Size & 1000 X 1000 \\
\hline Mac & $802.11 \mathrm{e}$ \\
\hline Radio Range & $250 \mathrm{~m}$ \\
\hline Simulation Time & $100 \mathrm{sec}$ \\
\hline Scheduler & EHAS \\
\hline Traffic Source & CBR \\
\hline Packet Size & 512 \\
\hline MSDU & 2132 \\
\hline Transmission Rate & $250 \mathrm{~Kb}$ \\
\hline No. of QSTAs & $5,10,15,20$ and 25 \\
\hline No. of sessions & $1,2,3,4$ and 5 \\
\hline
\end{tabular}

\subsection{Performance Metrics}

The performance is mainly evaluated according to: a) Average Ratio of Packet Delivery: It is the proportion of the total packets received to the total packets transmitted. $b$ ) Throughput: The average throughput received measured in $\mathrm{Mb} / \mathrm{sec}$, and c) Average End-to-End Delay: The average surviving data packets from source to destination.

\subsection{Results}

\subsubsection{Effect of Node Increase}

In the first simulation, the nodes are varied with an increment of 5 as $5,10,15,20$ and 25 .

Figure 1 illustrates the decrease in end-to-end delay when the MRCBQ protocol is used unlike the CLAE protocol, in the case of increased nodes. This is because of less traffic contention in case of MRCBQ.

MRCBQ achieves a good throughput and delivery ratio as it selects path with least signal fading and less traffic contention and there is a reduction in the average packet drop. Figure 2 illustrates this with the comparison of the average packet delivery ratio of MRCBQ and CLAE protocols.

Figure 3 demonstrates the increase in the throughput in the case of MRCBQ in comparison with CLAE when the nodes are increased.

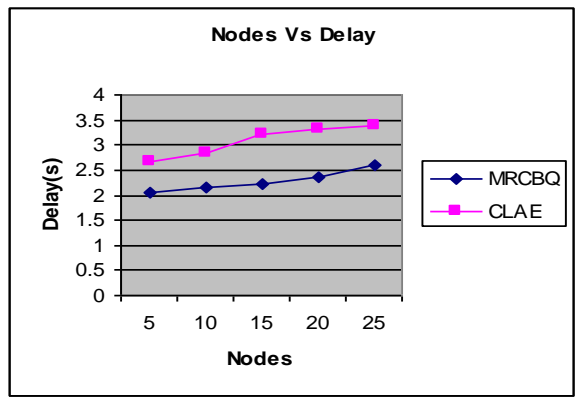

Fig 1: Nodes Vs Delay

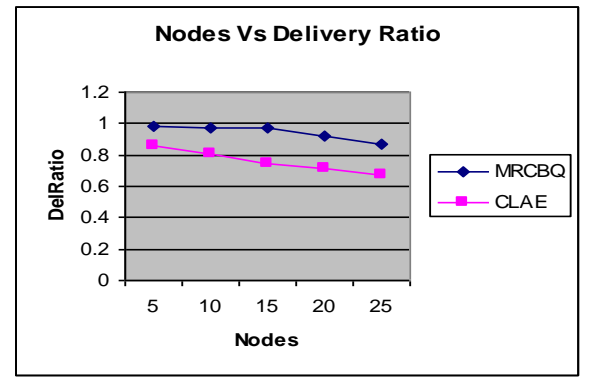

Fig2: Nodes Vs Delivery Ratio

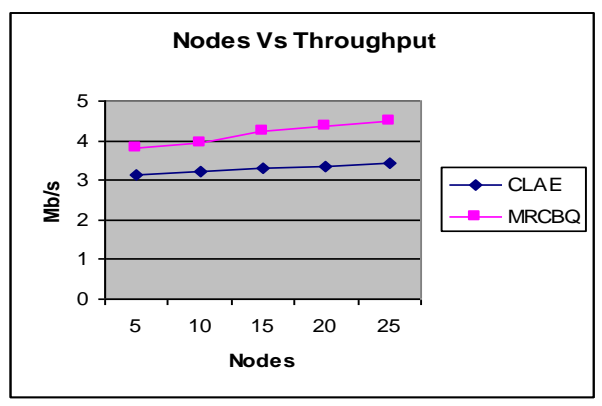

Fig 3: Nodes Vs Throughput

\subsubsection{Effect of varying Flows}

In the second experiment, the flows are varied as 1,2, 3, 4 and 5 .

Figure 4 indicates a decrease in end-to-end delay of flows when the MRCBQ protocol is used unlike the CLAE protocol, as MRCBQ selects the path with less traffic contention. Figure 5 indicates the better delivery ratio achieved by MRCBQ compared to CLAE protocol while figure 6 represents the increase in the throughput in the case of MRCBQ when compared to CLAE when there is an increase in the number of flows.

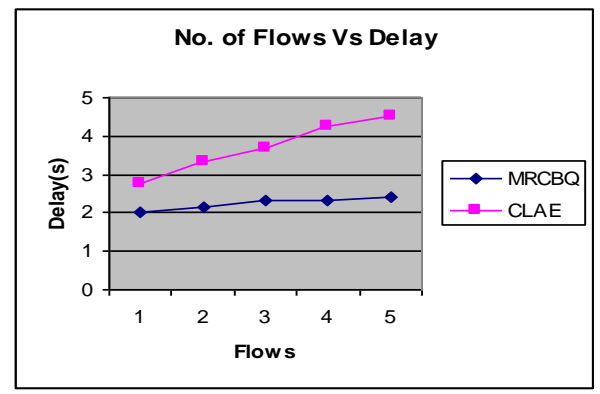

Fig 4: Flows Vs Delay

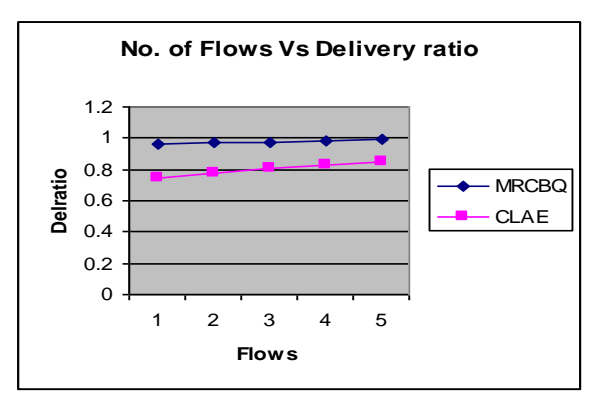

Fig 5: Flows Vs Delivery Ratio 


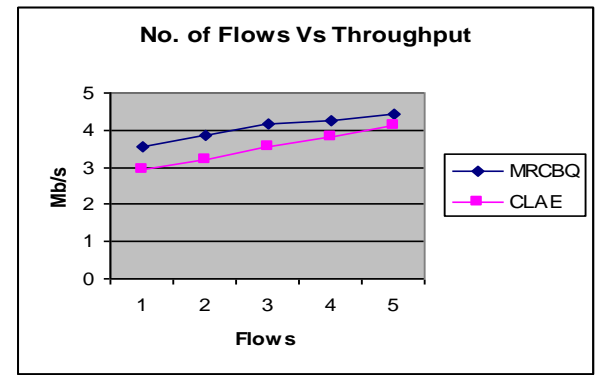

Fig 6: Flows Vs Throughput

\section{CONCLUSION}

A cross-layer based multipath routing protocol for IEEE 802.11e WLAN is suggested by the authors. Initially, multiple disjoint paths are determined followed by the estimation of a combined cost metric based on the Traffic Contention Time, Average Transmission Delay and Signal Fading Value. An RREQ is sent by the source $S$ to initiate the discovery of QoSaware route. The moment RREQ packet is received by $\mathrm{R} 1$, all metrics previously mentioned are computed and the combined cost CCR1 is calculated. R2 calculates its combined cost CCR 2 by adding the cost of R 1 and forwards the RREQ packet including the added cost. The RREQ will have all combined costs of all nodes by the time it arrives at the destination node D. The destination node D sends RREP, which includes all the node costs, to the source node $S$ via the intermediate nodes. On receiving the RREP from all the paths, the path with minimum combined cost is selected as the best path by the source $S$ and the data is transmitted through this path. The simulation results demonstrate that the proposed Multi-path routing protocol helps in achieving better delivery ratio and throughput with reduced delay.

\section{ACKNOWLEDGMENT}

This work is part of the first author's PhD work, which is supported under the Staff Development Program of Caledonian College of Engineering, Oman and National Institute of Technology Durgapur, India.

\section{REFERENCES}

[1] Shou-Chih Lo and W. T. Chen, "An Efficient Scheduling Mechanism for IEEE 802.11E MAC Enhancements," IEEE Wireless Communications and Networking Conference (WCNC), March 2004

[2] Muhammad Akber Farooqui, "Protocol Design Issues in WLAN", Journal of Lecture Notes in Engineering and Computer, VOL 2176, Issue 1, pages 279-282 (2009).

[3] Conroy Smith and Neco Ventura, "A MeasurementAided Model-Based Admission Control Scheme for IEEE 802.11e EDCA Wireless LANs," In Proceedings IFIP Symposium on Wireless Communications and Information Technology in Developing Countries ( WCITD), Oct. 2008.

[4] P. Ansel, Q. Ni, and T. Turletti, "An Efficient Scheduling Scheme for IEEE 802.11e," In Proceeding of Modeling and Optimization in Mobile, Ad Hoc and Wireless Networks, 2004.

[5] Y. Higuchi, A. Foronda, C. Ohta, M. Yoshimoto, and Y. Okada, "Delay guarantee and service interval optimization for HCCA in IEEE 802.11e WLANs," In Proceeding of IEEE Wireless Communications \& Networking Conference (WCNC 2007), 2007.

[6] Inanc.I, Keceli.F and Ayanoglu.E, "An Adaptive Multimedia QoS Scheduler for 802.11e Wireless LANs",
In Proceedings of the IEEE International Conference on communications, ICC-2006.

[7] Alan Marshall, "Quality of Service (QoS) for Wireless Networks", In the Proceeding of International Telecommunication union, October 2007.

[8] HCCA, http://en.wikipedia.org/wiki/IEEE_802.11e-2005

[9] Bo Han, Lusheng Ji, Seungjoon Lee, Robert R. Miller and Bobby Bhattacharjee, "Channel access throttling for improving WLAN QoS ", In Proceedings of the 6th Annual IEEE communications society conference on Sensor, Mesh and Ad Hoc Communications and Networks, 2009.

[10] Lamia Romdhani and Christian Bonnet, "A Cross-Layer On-Demand Routing Protocol for Delay-Sensitive Applications", 16th IEEE International Symposium on Personal Indoor and Mobile Radio Communications, 2005

[11] Xiaolin Cheng, Prasant Mohapatra, Sung-Ju Lee and Sujata Banerjee, "MARIA: Interference-Aware Admission Control and QoS Routing in Wireless Mesh Networks", IEEE ICC, 2008.

[12] Chris Barrett, Martin Drozda, Achla Marathe and Madhav V. Marathe, "Characterizing the Interaction between Routing and MAC Protocols in Adhoc Networks", Proceedings of the 3rd ACM international symposium on Mobile ad hoc networking \& computing, 2002.

[13] Ron Banner and Ariel Orda, "Multipath Routing Algorithms for Congestion Minimization", IEEE/ACM Transactions on Networking, 2007.

[14] Shafiullah Khan, Kok Keong Loo and Zia Ud Din, "Cross Layer Design for Routing and Security in Multihop Wireless Networks", Journal of Information Assurance and Security, 2009.

[15] Noureddine Kettaf, Hafid Abouaissa, Thang Vuduong and Pascal Lorenz, "A Cross layer Admission Control On-demand Routing Protocol for QoS Applications", IJCSNS International Journal of Computer Science and Network Security, 2006.

[16] Víctor Carrascal Frías, Guillermo Diaz Delgado and Monica Aguilar Igartua, "Multipath Routing for videostreaming services over IEEE 802.11e Ad hoc Networks", International Conference on Software in Telecommunications and Computer Networks, 2006. SoftCOM 2006.

[17] Carlos T. Calafate, Pietro Manzoni, and Manuel P. Malumbres, "On the interaction between IEEE 802.11e and routing protocols in Mobile Ad-hoc Networks", 13th IEEE Euromicro Conference on Parallel, Distributed and Network-Based Processing, 2005

[18] Hang Shen, Guangwei Bai, Junyuan Wang, Yong Jin and Jinjing Tao, "Performance Study of a Cross-Layer Based Multipath Routing Protocol for IEEE 802.11e Mobile Ad Hoc Networks", I. J. Communications, Network and System Sciences, 2008.

[19] Shaik Madhar Saheb, A. K. Bhattacharjee and A. Vallavaraj, "An Enhanced HCCA Based Adaptive Scheduler for Multimedia Traffic in IEEE 802.11e Wireless LAN", In: Proceedings of WORLDCOMP 10, ICWN 10, VOL 2, pages 446-453 (2010).

[20] Network Simulator: www.isi.edu/nsnam/ns 